\title{
Role of Monocyte Count and Neutrophil-to- Lymphocyte Ratio in Survival of Oral Cancer Patients
}

\author{
Saurabh Bobdey ${ }^{1}$ Balasubramaniam Ganesh ${ }^{1}$ Prabhashankar Mishra ${ }^{1}$ Aanchal Jain ${ }^{1}$ \\ ${ }^{1}$ Department of Medical Records, Biostatistics and Epidemiology, Tata \\ Memorial Hospital Parel, Mumbai, India \\ Address for correspondence Saurabh Bobdey, MD, DNB, Department \\ of Medical Records, Biostatistics and Epidemiology, Tata Memorial \\ Hospital, Parel, Mumbai 400012, India (e-mail: sbobdey@yahoo.com).
}

Int Arch Otorhinolaryngol 2017;21:21-27.

\begin{abstract}
Introduction Inflammation seems to play a critical role in the development and progression of numerous cancers. Peripheral blood leukocyte count is an easily assessable parameter of systemic inflammatory response.

Objective The aim of this study was to investigate whether the pretreatment leukocyte counts can predict the prognosis of patients with oral cavity cancer.

Methods Medical records of 471 oral cavity cancer patients diagnosed between January 2007 and December 2008 were retrospectively analyzed. Receiver operating characteristic curve analysis and Cox proportional hazards analyses were applied to evaluate the associations of leukocyte counts with overall survival.

Results The overall five year's survival of the cohort was found to be $49.4 \%$. On univariate analysis, elevated monocyte count $(\geq 500 / \mathrm{mm} 3)$ and neutrophil-to-lymphocyte ratio (NLR) (>2.38) were associated with poor overall survival (OS) $(p=0.001$ and 0.000 , respectively). Multivariate Cox proportional hazard analysis showed that higher

Keywords

- oral cancer

- prognosis

- survival

- monocyte

- neutrophil-tolymphocyte ratio monocyte and NLR levels were significant independent predictors of worse OS (HR $=1.385,95 \% \mathrm{Cl}=1.049-1.829 ; p<0.05$ and $\mathrm{HR}=1.392,95 \% \mathrm{Cl}=1.045-1.855$; $p<0.05$, respectively). The advanced overall stage and lymph nodal involvement were also independent indicators for poor OS.

Conclusions Higher pretreatment monocyte and NLR levels are independent predictors of poor prognosis for patients with oral cavity cancer. Thus, these easily accessed variables can serve as a potent marker to predict the outcomes of oral cancer patients.
\end{abstract}

\section{Introduction}

Oral cavity is one of the most frequent locations of squamous cell carcinoma in the head and neck region. Globally, an estimated 300,400 new cases of oral cavity cancer are diagnosed each year and it is the eleventh leading cause of cancer worldwide. In the Indian subcontinent oral cancer is a major public health problem and ranks among the top three types of cancer in the country. ${ }^{1}$ Almost 80,000 cases are diagnosed and 50,000 deaths occur annually due to oral cavity cancer in India. The 5-year survival rate for oral cancer in India is $\sim 37 \%$
(26-45). ${ }^{2}$ This indicates that, despite improvement in health infrastructure and treatment modalities, prognosis from oral cancer remains poor as compared with other Asian countries. ${ }^{2}$ Therefore, a low-cost, standardized, reliable, and reproducible prognostic marker for oral cancer patients is desirable to facilitate individualized treatments and, thus, better outcomes for the patients.

Inflammation seems to play a critical role in the development and progression of numerous cancers. ${ }^{3}$ Tumor development and progression induced by an inflammatory response is thought to be mediated by an interaction between received

February 17, 2016

accepted

June 18, 2016

published online

August 16, 2016
DOI http://dx.doi.org/

10.1055/s-0036-1587318. ISSN $1809-9777$.
Copyright $\odot 2017$ by Thieme-Revinter

Publicações Ltda, Rio de Janeiro, Brazil
License terms

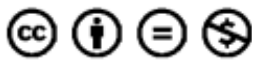


proinflammatory cytokines and pathways including nuclear factor-kappa B (NF-KB) and signal transducer \& activator of transcription 3 (STAT3). ${ }^{4}$ It has been postulated that the increased release of proinflammatory cytokines produces a systemic inflammatory response reflected in changes in circulating markers of inflammation, such as C-reactive protein and white blood cells. ${ }^{5}$ There are several lines of evidence to date suggesting that the total white blood cell count as well as its components, such as neutrophils, lymphocytes, monocytes, and the neutrophil-to-lymphocyte ratio (NLR) can predict survival in a variety of malignancies, including oral cavity, ${ }^{6,7}$ breast cancer, ${ }^{8}$ gastric cancer, ${ }^{9}$ hepatocellular carcinoma, ${ }^{10}$ Hodgkin's lymphoma ${ }^{11}$ and lung cancer. ${ }^{12}$ However, evidence for the use of hematologic markers of inflammation as predictors of clinical outcome in oral cavity cancer patients is limited. ${ }^{6,7}$ Given this background, the main objective of our study was to assess the role of total and differential leukocyte counts in overall survival of oral cavity cancer patients. The study had the approval of the Research Ethics Committee of the hospital.

\section{Methods}

We retrospectively analyzed the medical records of 471 pathologically proven oral cavity cancer patients, residents of Mumbai, diagnosed between January 01, 2007 to December 31,2008 , and who had not received any prior treatment. We retrospectively collected data from medical records on routine laboratory measurements of white blood cells (WBC) performed prior to onset of treatment, including the counts of neutrophils, lymphocytes, and monocytes. All patients were staged according to the sixth edition of the UICC/AJCC TNM classification system. ${ }^{13,14}$ In addition, we also collected data on age at diagnosis, sex, primary tumor site, and pretreatment tumor staging. We retrieved type of treatment received and status of the patient (Alive/ Dead) at the end of five years from the date of diagnosis from medical records.

Receiver operating characteristic (ROC) curve analysis to select the most appropriate cut-off points for the counts of total WBC, neutrophil, lymphocyte, and monocyte, and NLR was performed to stratify patients at a high risk of malignancyrelated death. We selected the score at the point with both maximum sensitivity and specificity as the best cut-off value. In survival analysis, overall survival time was defined as time from diagnosis until death; the follow-up of patients still alive has been censored at their latest date of follow-up. We generated survival curves by the Kaplan-Meier method and compared them by the log-rank test. We applied the Cox proportional hazards model for univariate and multivariate (backward method) analysis to identify prognostic factors. We performed statistical analyses using SPSS software v17.0 (SPSS, Chicago, IL).

\section{Results}

The patients' characteristics are summarized in - Table 1. As shown, the median age was 50 years (range: $25-85$ years), and the percentage of males and females were $69.9 \%$ and $30.1 \%$, respectively. Out of the 471 patients, 347 (73.67\%) were
Table 1 Patients' characteristics

\begin{tabular}{|c|c|c|}
\hline Parameter & No. of patients & Percentage (\%) \\
\hline \multicolumn{3}{|l|}{ Age } \\
\hline mean & 50 Years & - \\
\hline Range & 25-85 Years & - \\
\hline \multicolumn{3}{|l|}{ Sex } \\
\hline Male & 329 & 69.9 \\
\hline Female & 142 & 30.1 \\
\hline \multicolumn{3}{|c|}{ T Classification } \\
\hline T1 & 41 & 8.7 \\
\hline $\mathrm{T} 2$ & 142 & 30.1 \\
\hline T3 & 56 & 11.9 \\
\hline $\mathrm{T} 4$ & 232 & 49.3 \\
\hline \multicolumn{3}{|c|}{ N Classification } \\
\hline No & 208 & 44.2 \\
\hline $\mathrm{N}+$ & 263 & 55.8 \\
\hline \multicolumn{3}{|c|}{ M Classification } \\
\hline M0 & 469 & 99.6 \\
\hline M1 & 2 & 0.4 \\
\hline \multicolumn{3}{|c|}{ Tumor Stage, AJCC } \\
\hline I & 32 & 6.8 \\
\hline II & 92 & 19.5 \\
\hline III & 76 & 16.1 \\
\hline IV & 271 & 57.5 \\
\hline
\end{tabular}

Abbreviations: AJCC, American Joint Committee on Cancer.

diagnosed at late stages (III and IV) and the other 124 patients (26.33\%) were at early stages (I and II). The median follow-up period was 22 months (range, 0 to 98 months). The five-year overall survival of the cohort was $49.4 \%$ and stage-wise survival rate for TNM stage I, II, III, and IV patients was found to be $86.4 \%, 68 \%, 57.9 \%$, and $33.6 \%$ ( $p<0.00$ ), respectively.

We determined the cut-off value of total WBC, neutrophil, lymphocyte, monocyte counts, and neutrophil lymphocyte ratio (NLR) for survival outcomes by ROC curve analyses. We selected the NLR cut-off point of 2.38 for the survival analyses and divided all patients into either high (NLR $\geq 2.38$ ) or low (NLR $<2.38$ ) NLR groups. Additionally, to ensure comparability with other studies, we also conducted an analysis using NLR cut-off of 5; however, only 72 (15.3\%) patients had NLR of $\leq 5$. Similarly, we selected as the optimal cut-off points for survival analyses, a total WBC count of $7.900 \times 10^{9} / \mathrm{L}$, neutrophil count of $4.900 \times 10^{9} / \mathrm{L}$, lymphocyte count of $1.980 \times 10^{9} / \mathrm{L}$, and monocyte count of $0.500 \times 10^{9} / \mathrm{L}$.

We performed a univariate analysis of age, gender, overall stage, lymph node involvement, treatment modality, total WBC count, neutophil count, lymphocyte count, monocyte count, and NLR to assess their role as prognostic factors for overall survival. Univariate analysis revealed that lower WBC count $\left(<7.900 \times 10^{9} / \mathrm{L} ; p=0.026\right)$, monocyte count $\left(<0.500 \times 10^{9} / \mathrm{L} ; p=0.001\right)$, and NLR level 
Table 2 Univariate and multivariate analysis of prognostic factors for overall survival in patients with oral cavity

\begin{tabular}{|c|c|c|c|c|}
\hline \multirow[b]{2}{*}{ Parameter } & \multicolumn{2}{|l|}{ Univariate } & \multicolumn{2}{|l|}{ Multivariate } \\
\hline & $\begin{array}{l}\mathrm{HR} \\
(95 \% \mathrm{Cl})\end{array}$ & $\mathrm{p}$ & $\begin{array}{l}\text { HR } \\
(95 \% \mathrm{Cl})\end{array}$ & $\mathrm{p}$ \\
\hline Age, $\geq 65$ years & $\begin{array}{l}1.088 \\
(0.745-1.589)\end{array}$ & 0.664 & - & - \\
\hline Sex, (male) & $\begin{array}{l}1.244 \\
(0.928-1.669)\end{array}$ & 0.145 & - & - \\
\hline TNM stage (III + IV) & $\begin{array}{l}3.155 \\
(2.168-4.591)\end{array}$ & $0.000^{* *}$ & $\begin{array}{l}1.921 \\
(1.193-3.092)\end{array}$ & $0.007^{* *}$ \\
\hline Lymph node mets & $\begin{array}{l}2.492 \\
(1.876-3.310)\end{array}$ & $0.000^{* *}$ & $\begin{array}{l}1.715 \\
(1.200-2.451)\end{array}$ & $0.003^{* *}$ \\
\hline Neutrophil $\geq 4.90010^{9} / \mathrm{L}$ & $\begin{array}{l}1.290 \\
(0.989-1.683)\end{array}$ & 0.060 & - & - \\
\hline Lymphocyte $\leq 1.98010^{9} / \mathrm{L}$ & $\begin{array}{l}1.224 \\
(0.941-1.592)\end{array}$ & 0.131 & - & - \\
\hline Monocyte $\geq 0.50010^{9} / \mathrm{L}$ & $\begin{array}{l}1.558 \\
(1.190-2.040) \\
\end{array}$ & $0.001^{* *}$ & $\begin{array}{l}1.385 \\
(1.049-1.829)\end{array}$ & $0.021^{* *}$ \\
\hline $\mathrm{WBC} \geq 7.90010^{9} / \mathrm{L}$ & $\begin{array}{l}1.352 \\
(1.037-1.764)\end{array}$ & $0.026^{* *}$ & & 0.164 \\
\hline Neutrophil/ lymphocyte ratio (NLR), $\geq 2.38$ & $\begin{array}{l}1.676 \\
(1.271-2.209)\end{array}$ & $0.000^{* *}$ & $\begin{array}{l}1.392 \\
(1.045-1.855)\end{array}$ & $0.024^{* *}$ \\
\hline Neutrophil/ lymphocyte ratio (NLR), $\geq 5$ & $\begin{array}{l}1.348 \\
(0.954-1.904)\end{array}$ & 0.091 & - & - \\
\hline Treatment (single/ multi-modality) & $\begin{array}{l}0.924 \\
(0.712-1.200)\end{array}$ & 0.555 & - & - \\
\hline
\end{tabular}

Abbreviations: $\mathrm{Cl}$, confidence interval; $\mathrm{HR}$, hazard ratio. ${ }^{* *}$ Significant $(\mathrm{p}<0.05)$.

$(<2.38 ; p<0.001)$ were associated with superior survival, but when we used traditional NLR cut-off of 5, we did not find it to be significantly associated with survival $(p=0.091)$. In addition, advanced disease (TNM stage, III + IV; $\quad p<0.001)$ and lymph nodal involvement $(p<0.001)$ were found to be associated with poorer prognosis. Other factors such as age, gender, neutrophil count, lymphocyte count, and treatment modality were not significantly associated with survival of oral cavity cancer patients.

We included all the characteristics, such as age, gender, overall stage, lymph node involvement, treatment modality, total WBC count, neutophil count, lymphocyte count, monocyte count, and NLR in the multivariate analysis. The results showed that higher NLR level $(\mathrm{HR}=1.392,95 \% \mathrm{CI}=1.045-1.855$; $p<0.001)$, monocyte count $(\mathrm{HR}=1.385,95 \% \mathrm{CI}=1.049$ $1.829 ; p<0.001)$, advanced stage disease (HR $=1.921,95 \%$ $\mathrm{CI}=1.193-3.092 ; p<0.001)$, and lymph node involvement $(\mathrm{HR}=1.715,95 \% \mathrm{CI}=1.200-2.451 ; p<0.001)$ were independent predictors for poor overall survival of oral cavity cancer patients (-Table 2 ).

The five-year survival rate of patients with high monocyte count $\left(\geq 0.500 \times 10^{9} / \mathrm{L}\right.$ ) was found to be $43.9 \%$, which was significantly ( $p<0.001$ ) less than the $55.4 \%$ survival rate for patients with lower monocyte count (-Fig. 1). Similarly, we found five yearś survival rate for patients with higher $(\geq 2.38)$ and lower NLR was $42.2 \%$ and $58.6 \%$, respectively $(\boldsymbol{p}<0.001)$ ( - Fig. 2). We categorized patient characteristics including hematological parameters as high / low NLR and monocyte counts (-Table 3). Patients with high NLR level ( $\geq 2.38$ ) had a higher incidence of advanced $T, N$, and overall stages $(\mathrm{p}=0.001, p=0.015$, and $p=0.003$, respectively). Lifestyle factors such as tobacco chewing, smoking and alcohol consumption did not appear to be associated with NLR or monocyte count ( $p>0.05)$.

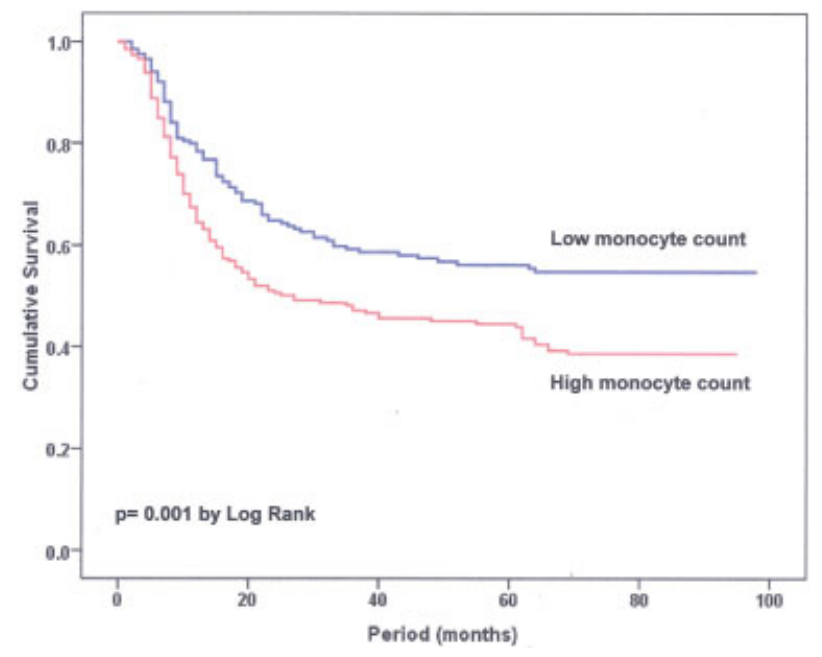

Fig. 1 Kaplan-Meier survival curves of cumulative survival rates in patients with oral cavity cancer classified into two groups according to monocyte count. 


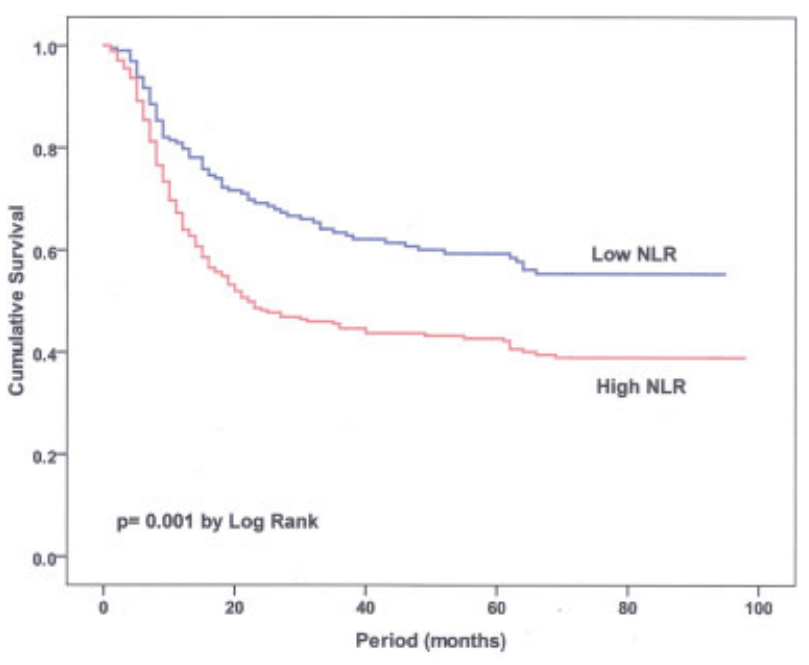

Fig. 2 Kaplan-Meier survival curves of cumulative survival rates in patients with oral cavity cancer classified into two groups according to NLR.

\section{Discussion}

The total and differential white blood cell (WBC) count has been historically used as a marker of infection and inflammation. Nonetheless, its role has gone beyond the assessment of infectious processes and it has become an important prognostic measurement of outcomes in cancer treatment. Thus, while a link between inflammation and cancer has been known for more than a century, compelling recent evidence have suggested a strong association between pretreatment peripheral inflammatory cells and prognosis in different kinds of cancers. ${ }^{6-12}$ As part of the functional relevance, inflammatory responses lead to chronic oxidative stress and generate oxygen free radicals, which have been shown to stimulate cancer initiation, promotion, and progression. ${ }^{15,16}$ Here, we have performed a retrospective medical record based study on oral cavity cancer to evaluate the prognostic values of total WBC, neutrophil, lymphocyte, monocyte counts, and NLR together with the other clinical factors. Our results confirmed the previous findings that factors such as absence of nodal involvement and early stage, were associated with favorable prognosis for oral cavity cancer patients. ${ }^{17,18}$ More importantly, we found that an elevated neutrophil lymphocyte ratio (NLR) and monocyte count were significantly associated with poorer overall survival and were independent of other variables to predict the prognosis for oral cavity cancer patients. These results were in consonance with other published studies that implicate role of monocyte count (Tsai et al, $n=213)^{6}$ and NLR (Perisanidis et al, $n=97)^{7}$ in prognostication of oral cavity cancer patients. However, these studies relied on a relatively smaller sample and the cut-off value for monocyte count was based on a median value of circulating monocyte count. In the present study, to exclude empirical bias, we used ROC curve to determine the optimal cut-off value to predict risk of malignancy related death.

In the present study, we found that monocyte count was an independent prognostic factor for patients with oral cavity cancer. There is substantial evidence that, in advanced cancer, the host systemic immune response is an important independent predictor of outcome, and that pretreatment measurements of the systemic inflammatory immune response can be used to independently predict cancer survival. ${ }^{6,19}$ Sasaki and colleagues ${ }^{20,21}$ studied the pre-operative absolute monocyte count in patients who had liver resection due to hepatocellular carcinoma, as well as in patients who underwent hepatic surgery due to colorectal metastasis and found that pretreatment absolute monocyte count was an independent prognostic indicator of tumor recurrence and survival in patients with hepatocellular carcinoma. Similarly, absolute monocyte count has been reported to be independent prognostic indicator for breast cancer, ${ }^{8}$ gastric cancer, ${ }^{9}$ Hodgkin's lymphoma, ${ }^{11}$ Colorectal cancer, ${ }^{21}$ and Ovarian cancer. ${ }^{22}$

The exact underlying mechanism explaining the association between the elevated number of monocytes and unfavorable cancer prognosis is unclear. However, a possible explanation can be that monocytes secrete various proinflammatory cytokines, such as interleukin (IL)-1, IL-6, IL-10, and TNF- $\alpha$, which have been associated with shorter survival and worse prognosis in malignances. $^{23,24}$ Moreover, monocytes upon stimulation are known to release monocyte chemo-attractant protein (MCP-1)-1 and mediate tumor-associated macrophage infiltration in solid tumors, which could produce a variety of chemokines such as TGF- $\alpha$, TNF- $\alpha$, IL-1, and IL-6 to promote tumorigenesis, angiogenesis, and distant metastasis of malignant tumors. ${ }^{24,25}$ Further, studies have linked monocyte with an increased number of bone marrow-derived myelomonocytic cells. These cells infiltrate the tumor and differentiate into tumor-associated macrophages, which in turn release many angiogenic factors and have been shown to be associated with poor prognosis in cancers. ${ }^{24,26,27}$

The other main finding of our analysis was that high pretreatment NLR was significantly associated with poor survival in oral cavity cancer patients. This result is in accordance with previous observations on the association between NLR and a variety of cancers such as lung cancer, colorectal cancer, cholangiocarcinoma, and Pancreatic cancer. $^{28-32}$ More specifically, in Head and Neck cancers, elevated pretreatment NLR has been shown to be significantly associated with worse survival in two studies of nasopharyngeal cancer patients ${ }^{33,34}$ and only one study of oral cavity cancer patients. ${ }^{7}$ In all three studies, NLR cut-off was taken on the basis of either median value or ROC analysis and none of them have considered traditional NLR cut-off of 5.,33,34 In our study, oral cancer survival was also found to be associated with an ROC-based NLR cut-off of 2.38, but failed to achieve statistical significance with the traditional NLR cut-off of 5 . This can be due to the small sample size, from which few patients had an NLR above 5 .

Thus, to establish a ROC-based lower NLR cut-off for predicting survival in oral cancer patients larger, multicenter prospective studies are needed. In our study, we also assessed the prognostic value of the individual components of NLR, that is, neutrophil and lymphocyte count. Individually, however, neither was significantly associated with survival of oral cavity cancer patients. Similarly, Perisanidis et al also reported a significant 
Table 3 Baseline clinical characteristics of the 471 Oral cavity carcinoma patients according to high/ low Neutrophil lymphocyte ratio (NLR) and monocyte count

\begin{tabular}{|c|c|c|c|c|c|c|}
\hline Characteristic & $\begin{array}{l}\text { NLR }<2.38 \\
(n=199)\end{array}$ & $\begin{array}{l}\text { NLR } \geq 2.38 \\
(n=272)\end{array}$ & $p$ & $\begin{array}{l}\text { LM Count } \\
<500\left(10^{9} / \mathrm{L}\right) \\
(n=206)\end{array}$ & $\begin{array}{l}\text { HM Count } \\
\geq 500\left(10^{9} / \mathrm{L}\right) \\
(n=265)\end{array}$ & $\mathrm{p}$ \\
\hline Mean age (years) & $50.20 \pm 12.01$ & $51.84 \pm 12.48$ & 0.152 & $50.37 \pm 11.53$ & $51.75 \pm 12.85$ & 0.225 \\
\hline \multicolumn{7}{|l|}{ Tobacco, no. (\%) } \\
\hline Non-chewers & $82(41.2)$ & $120(44.1)$ & 0.528 & $92(44.7)$ & $110(41.5)$ & 0.493 \\
\hline Chewers & $117(58.8)$ & $152(55.9)$ & - & $114(55.3)$ & $155(58.5)$ & - \\
\hline Non-smokers & $144(72.4)$ & $212(77.9)$ & 0.164 & $153(74.3)$ & $203(76.6)$ & 0.559 \\
\hline Smokers & $55(27.6)$ & $60(22.1)$ & - & $53(25.7)$ & $62(23.4)$ & - \\
\hline \multicolumn{7}{|l|}{ Alcohol, no. (\%) } \\
\hline Non-drinker & $156(78.4)$ & $229(84.2)$ & 0.108 & $171(83.0)$ & $214(80.8)$ & 0.530 \\
\hline Drinker & $43(21.6)$ & $43(15.8)$ & - & $35(17.0)$ & $51(19.2)$ & - \\
\hline \multicolumn{7}{|l|}{ T-classification } \\
\hline $\mathrm{T} 1-\mathrm{T} 2$ & $105(52.8)$ & $78(28.7)$ & $0.000^{* *}$ & $86(47.0)$ & $97(53.0)$ & 0.256 \\
\hline T3-T4 & $94(47.2)$ & $194(71.3)$ & - & $120(58.3)$ & $168(63.4)$ & - \\
\hline \multicolumn{7}{|l|}{$\mathrm{N}$-classification } \\
\hline No & $101(50.8)$ & $107(39.5)$ & $0.015^{* *}$ & $99(48.1)$ & $109(41.3)$ & 0.143 \\
\hline $\mathrm{N}+$ & $98(49.2)$ & $164(60.5)$ & - & $107(51.9)$ & $155(58.7)$ & - \\
\hline \multicolumn{7}{|l|}{ Overall stage } \\
\hline$|-| \mid$ & $75(37.7)$ & $49(18.0)$ & $0.000^{* *}$ & $63(30.6)$ & $61(23.0)$ & 0.064 \\
\hline III-IV & $124(62.3)$ & $223(82.0)$ & - & $143(69.4)$ & $204(77.0)$ & - \\
\hline \multicolumn{7}{|l|}{ Treatment } \\
\hline Uni-modality & $98(49.2)$ & $127(46.7)$ & 0.583 & $100(48.5)$ & $125(47.2)$ & 0.767 \\
\hline Dual/ Multi-modality & $101(50.8)$ & $145(53.3)$ & - & $106(51.5)$ & $140(52.8)$ & - \\
\hline \multicolumn{7}{|c|}{ Hematological Parameters } \\
\hline Red blood cell, X10 /IL & $4.60 \pm 0.64$ & $4.57 \pm 0.70$ & 0.145 & $4.52 \pm 0.69$ & $4.53 \pm 0.67$ & 0.888 \\
\hline Hemoglobin, g/dL & $12.8 \pm 2.1$ & $12.7 \pm 2.2$ & 0.528 & $12.7 \pm 2.2$ & $12.7 \pm 2.2$ & 0.987 \\
\hline Hematocrit, \% & $38.6 \pm 5.9$ & $38.2 \pm 6.4$ & 0.464 & $38.3 \pm 6.2$ & $38.4 \pm 6.2$ & 0.884 \\
\hline $\mathrm{MCV}, \mathrm{fL}$ & $84.4 \pm 10.0$ & $85.8 \pm 9.6$ & 0.144 & $85.1 \pm 9.3$ & $85.2 \pm 10.1$ & 0.925 \\
\hline $\mathrm{MCH}, \mathrm{pg} / \mathrm{cell}$ & $28.0 \pm 3.8$ & $28.5 \pm 3.7$ & 0.165 & $28.3 \pm 3.7$ & $28.3 \pm 3.9$ & 0.994 \\
\hline $\mathrm{MCHC}, \mathrm{g} / \mathrm{dL}$ & $33.1 \pm 1.3$ & $33.2 \pm 1.3$ & 0.688 & $33.2 \pm 1.3$ & $33.1 \pm 1.3$ & 0.640 \\
\hline
\end{tabular}

Abbreviations: HM, high monocyte; LM, low monocyte; MCH, mean corpuscular hemoglobin; MCHC, mean corpuscular-hemoglobin concentration; $\mathrm{MCV}$, mean corpuscular volume. $\mp$ Data are means $\pm S D$; ${ }^{* *}$ Significant $(p<0.05)$.

relationship between NLR and oral cancer survival but not with its individual components. ${ }^{7}$ It has been suggested that, in cancer patients, NLR is superior to other individual leukocyte parameters. $^{35}$ This superiority of NLR can be attributed to the stability of NLR compared with the absolute counts that could be altered by various physiological, pathological, and physical factors. Moreover, NLR may represent the two opposing inflammatory and immune pathways that exist together in cancer patients. ${ }^{7}$ Therefore, NLR can be considered as the balance between protumor inflammatory status and anti-tumor immune status. Patients with elevated NLR have a relative neutrophilic leukocytosis and lymphocytopenia, which denotes that the balance is inclined in favor of pro-tumor inflammatory and is associated with poor outcome. ${ }^{34,35}$
The mechanisms underlying the association of high NLR and poor outcome of cancer patients are poorly understood. One potential mechanism underlying the prognostic impact of NLR may be an association of high NLR with inflammation. An elevated NLR has been associated with an increase in the peritumoral infiltration of macrophages and an increase in interleukin (IL-17). ${ }^{36}$ Neutrophils and other cells such as macrophages have been reported to secrete tumor growth promoting factors, including vascular endothelial growth factor, ${ }^{37}$ hepatocyte growth factor, ${ }^{38}$ IL-6, ${ }^{39}$ IL-8, ${ }^{40}$ matrix metalloproteinases, ${ }^{41}$ and elastases, ${ }^{42}$ and, thus, likely contribute to a stimulating tumor microenvironment. It is a consensus that the adaptive immune system carries out immune surveillance and can eliminate newborn tumors, 
but effective adaptive immune responses are always suppressed in established tumors through several pathways, including inhibition of dendritic cell differentiation and activation, infiltration of regulatory T cells. ${ }^{43}$ Lymphocytes are crucial components of adaptive immune system, and studies have reported infiltrating lymphocytes to indicate the generation of an effective antitumor cellular immune response. ${ }^{44} \mathrm{~A}$ low peripheral lymphocyte level may indicate a poorer lymphocyte-mediated immune response to tumor and suggests poor prognosis. ${ }^{45,46}$ NLR may be explained by the diverse effects of neutrophils and lymphocytes on tumor progression. In vitro studies have shown that the cytolytic activity of lymphocytes and natural killer cells was suppressed when cocultured with neutrophils, and the extent of suppression was proportionally enhanced to the addition of neutrophils, ${ }^{46-48}$ implying that high NLR was associated with poor prognosis. ${ }^{33}$

\section{Conclusion}

We found that both NLR and monocyte counts are independent predictors of overall survival for patients with oral cavity cancer. Given the low cost, easy accessibility, and reproducibility of a full blood count, both NLR and monocyte counts seem promising candidates for use in clinical practice. However, the findings of the study are based on a retrospective design in a single center, thus, further studies in either multicenter or prospective manner should be undertaken to validate and determine the clinical usages of NLR and monocyte count as prognostic markers for oral cavity cancer patients.

\section{Conflict of Interest}

The authors declare no conflict of interest in conducting this study.

\section{References}

1 Ferlay J, Shin HR, Bray F, Forman D, Mathers C, Parkin DM. GLOBOCAN 2012, Cancer incidence and mortality worldwide: IARC CancerBase No. 10. Lyon, France: International Agency for Research on Cancer; 2012. Available at http://globocan.iarc.fr. Accessed on Dec 12, 2015

2 Sankaranarayanan R, Swaminathan R, Lucas E. Cancer survival in Africa, Asia, Caribbean and Central America: SURVCAN. Lyon, France: IARC Scientific publication international agency for research on cancer; 2010

3 Weitzman SA, Gordon LI. Inflammation and cancer: role of phagocyte-generated oxidants in carcinogenesis. Blood 1990;76(4): 655-663

4 Jarnicki A, Putoczki T, Ernst M. Stat3: linking inflammation to epithelial cancer: More than a "gut" feeling? Cell Div 2010;5:14

5 Kao SC, Pavlakis N, Harvie R, et al. High blood neutrophil-tolymphocyte ratio is an indicator of poor prognosis in malignant mesothelioma patients undergoing systemic therapy. Clin Cancer Res 2010;16(23):5805-5813

6 Tsai YD, Wang CP, Chen CY, et al. Pretreatment circulating monocyte count associated with poor prognosis in patients with oral cavity cancer. Head Neck 2014;36(7):947-953
7 Perisanidis C, Kornek G, Pöschl PW, et al. High neutrophil-tolymphocyte ratio is an independent marker of poor diseasespecific survival in patients with oral cancer. Med Oncol 2013; 30(1):334-335

8 Hornychova H, Melichar B, Tomsova M, Mergancova J, Urminska H, Ryska A. Tumor-infiltrating lymphocytes predict response to neoadjuvant chemotherapy in patients with breast carcinoma. Cancer Invest 2008;26(10):1024-1031

9 Bruckner HW, Lavin PT, Plaxe SC, Storch JA, Livstone EM. Absolute granulocyte, lymphocyte, and moncyte counts. Useful determinants of prognosis for patients with metastatic cancer of the stomach. JAMA 1982;247(7):1004-1006

10 Chew V, Chen J, Lee D, et al. Chemokine-driven lymphocyte infiltration: an early intratumoural event determining longterm survival in resectable hepatocellular carcinoma. Gut 2012; 61(3):427-438

11 Koh YW, Kang HJ, Park C, et al. The ratio of the absolute lymphocyte count to the absolute monocyte count is associated with prognosis in Hodgkin's lymphoma: correlation with tumor-associated macrophages. Oncologist 2012;17(6):871-880

12 Tibaldi C, Vasile E, Bernardini I, Orlandini C, Andreuccetti M, Falcone A. Baseline elevated leukocyte count in peripheral blood is associated with poor survival in patients with advanced nonsmall cell lung cancer: a prognostic model. J Cancer Res Clin Oncol 2008;134(10):1143-1149

13 Greene FL. The American Joint Committee on Cancer: updating the strategies in cancer staging. Bull Am Coll Surg 2002;87(7): $13-15$

14 Greene FL, Sobin LH. The staging of cancer: a retrospective and prospective appraisal. CA Cancer J Clin 2008;58(3):180-190

15 Nakamura Y, Gindhart TD, Winterstein D, Tomita I, Seed JL, Colburn NH. Early superoxide dismutase-sensitive event promotes neoplastic transformation in mouse epidermal JB6 cells. Carcinogenesis 1988;9(2):203-207

16 Hussain SP, Aguilar F, Amstad P, Cerutti P. Oxy-radical induced mutagenesis of hotspot codons 248 and 249 of the human p53 gene. Oncogene 1994;9(8):2277-2281

17 Baatenburg de Jong RJ, Hermans J, Molenaar J, Briaire JJ, le Cessie S. Prediction of survival in patients with head and neck cancer. Head Neck 2001;23(9):718-724

18 Sayed SI, Sharma S, Rane P, et al. Can metastatic lymph node ratio (LNR) predict survival in oral cavity cancer patients? J Surg Oncol 2013;108(4):256-263

19 Roxburgh CS, McMillan DC. Role of systemic inflammatory response in predicting survival in patients with primary operable cancer. Future Oncol 2010;6(1):149-163

20 Sasaki A, Iwashita Y, Shibata K, Matsumoto T, Ohta M, Kitano S. Prognostic value of preoperative peripheral blood monocyte count in patients with hepatocellular carcinoma. Surgery 2006;139(6): 755-764

21 Sasaki A, Kai S, Endo Y, et al. Prognostic value of preoperative peripheral blood monocyte count in patients with colorectal liver metastasis after liver resection. J Gastrointest Surg 2007;11(5): 596-602

22 Bishara S, Griffin M, Cargill A, et al. Pre-treatment white blood cell subtypes as prognostic indicators in ovarian cancer. Eur J Obstet Gynecol Reprod Biol 2008;138(1):71-75

23 Anand M, Chodda SK, Parikh PM, Nadkarni JS. Abnormal levels of proinflammatory cytokines in serum and monocyte cultures from patients with chronic myeloid leukemia in different stages, and their role in prognosis. Hematol Oncol 1998; 16(4):143-154

24 Pollard JW. Tumour-educated macrophages promote tumour progression and metastasis. Nat Rev Cancer 2004;4(1):71-78

25 Hefler L, Tempfer C, Heinze G, et al. Monocyte chemoattractant protein-1 serum levels in ovarian cancer patients. $\mathrm{Br} \mathrm{J}$ Cancer 1999;81(5):855-859 
26 Dirkx AE, Oude Egbrink MG, Wagstaff J, Griffioen AW. Monocyte/ macrophage infiltration in tumors: modulators of angiogenesis. J Leukoc Biol 2006;80(6):1183-1196

27 Lewis CE, Pollard JW. Distinct role of macrophages in different tumor microenvironments. Cancer Res 2006;66(2):605-612

28 Halazun KJ, Aldoori A, Malik HZ, et al. Elevated preoperative neutrophil to lymphocyte ratio predicts survival following hepatic resection for colorectal liver metastases. Eur J Surg Oncol 2008;34(1):55-60

29 Walsh SR, Cook EJ, Goulder F, Justin TA, Keeling NJ. Neutrophillymphocyte ratio as a prognostic factor in colorectal cancer. J Surg Oncol 2005;91(3):181-184

30 Gomez D, Morris-Stiff G, Toogood GJ, Lodge JP, Prasad KR. Impact of systemic inflammation on outcome following resection for intrahepatic cholangiocarcinoma. J Surg Oncol 2008;97(6):513-518

31 Sarraf KM, Belcher E, Raevsky E, Nicholson AG, Goldstraw P, Lim E. Neutrophil/lymphocyte ratio and its association with survival after complete resection in non-small cell lung cancer. J Thorac Cardiovasc Surg 2009;137(2):425-428

32 Wang DS, Luo HY, Qiu MZ, et al. Comparison of the prognostic values of various inflammation based factors in patients with pancreatic cancer. Med Oncol 2012;29(5):3092-3100

33 He JR, Shen GP, Ren ZF, et al. Pretreatment levels of peripheral neutrophils and lymphocytes as independent prognostic factors in patients with nasopharyngeal carcinoma. Head Neck 2012; 34(12):1769-1776

34 An X, Ding PR, Wang FH, Jiang WQ Li YH. Elevated neutrophil to lymphocyte ratio predicts poor prognosis in nasopharyngeal carcinoma. Tumour Biol 2011;32(2):317-324

35 Azab B, Bhatt VR, Phookan J, et al. Usefulness of the neutrophil-tolymphocyte ratio in predicting short- and long-term mortality in breast cancer patients. Ann Surg Oncol 2012;19(1):217-224

36 Motomura T, Shirabe K, Mano Y, et al. Neutrophil-lymphocyte ratio reflects hepatocellular carcinoma recurrence after liver transplantation via inflammatory microenvironment. J Hepatol 2013;58(1):58-64

37 McCourt M, Wang JH, Sookhai S, Redmond HP. Proinflammatory mediators stimulate neutrophil-directed angiogenesis. Arch Surg 1999;134(12):1325-1331, discussion 1331-1332
38 McCourt M, Wang JH, Sookhai S, Redmond HP. Activated human neutrophils release hepatocyte growth factor/scatter factor. Eur J Surg Oncol 2001;27(4):396-403

39 Jabłońska E, Kiluk M, Markiewicz W, Piotrowski L, Grabowska Z, Jabłoński J. TNF-alpha, IL-6 and their soluble receptor serum levels and secretion by neutrophils in cancer patients. Arch Immunol Ther Exp (Warsz) 2001;49(1):63-69

40 Schaider H, Oka M, Bogenrieder T, et al. Differential response of primary and metastatic melanomas to neutrophils attracted by IL-8. Int J Cancer 2003;103(3):335-343

41 Shamamian P, Schwartz JD, Pocock BJ, et al. Activation of progelatinase A (MMP-2) by neutrophil elastase, cathepsin $G$, and proteinase-3: a role for inflammatory cells in tumor invasion and angiogenesis. J Cell Physiol 2001;189(2): 197-206

42 Scapini P, Nesi L, Morini M, et al. Generation of biologically active angiostatin kringle $1-3$ by activated human neutrophils. J Immunol 2002;168(11):5798-5804

43 Mantovani A, Allavena P, Sica A, Balkwill F. Cancer-related inflammation. Nature 2008;454(7203):436-444

44 Rabinowich H, Cohen R, Bruderman I, Steiner Z, Klajman A. Functional analysis of mononuclear cells infiltrating into tumors: lysis of autologous human tumor cells by cultured infiltrating lymphocytes. Cancer Res 1987;47(1):173-177

45 Cho H, Hur HW, Kim SW, et al. Pre-treatment neutrophil to lymphocyte ratio is elevated in epithelial ovarian cancer and predicts survival after treatment. Cancer Immunol Immunother 2009;58(1):15-23

46 Yamanaka T, Matsumoto S, Teramukai S, Ishiwata R, Nagai Y, Fukushima M. The baseline ratio of neutrophils to lymphocytes is associated with patient prognosis in advanced gastric cancer. Oncology 2007;73(3Y4):215-220

47 Petrie HT, Klassen LW, Kay HD. Inhibition of human cytotoxic T lymphocyte activity in vitro by autologous peripheral blood granulocytes. J Immunol 1985;134(1):230-234

48 el-Hag A, Clark RA. Immunosuppression by activated human neutrophils. Dependence on the myeloperoxidase system. J Immunol 1987;139(7):2406-2413 